\title{
Effect of a hypercholesterolemia as a starting factor on spinal degeneration in rabbits and role of Vitamin E (a-tocopherol)
}

$\underline{\text { Mehdi Sasani, }},{ }^{*}$ Ahmet Levent Aydın, ${ }^{1}$ Nurgul Aytan, ${ }^{2}$ Ozlem Yapicier, ${ }^{3}$ Tunc Oktenoglu, $\underline{\text { Nesrin Kartal Ozer, }}{ }^{4}$ and Ali Fahir Ozer ${ }^{5}$

Department of Neurosurgery, American Hospital, Istanbul, Turkey, Turkey

${ }^{1}$ Department of Neurosurgery, Istanbul Physical Therapy and Rehabilitation Training Hospital, Istanbul, Turkey

${ }^{2}$ Department of Neurology, School of Medicine, Boston University, Boston, MA, USA

${ }^{3}$ Department of Pathology, School of Medicine, Acibadem University, Istanbul, Turkey

${ }^{4}$ Department of Biochemistry, Faculty of Medicine, Marmara University, Istanbul, Turkey

5 Department of Neurosurgery, School of Medicine, Koc University, Istanbul, Turkey

Mehdi Sasani: sasanim@gmail.com; Ahmet Levent Aydın: ahmetleventaydin@yahoo.com; Nurgul Aytan: naytan@bu.edu; Ozlem Yapicier: ozlemyapicier@yahoo.com; Tunc Oktenoglu: tuncoktenoglu@gmail.com; Nesrin Kartal Ozer: nkozer@marmara.edu.tr; Ali Fahir Ozer: alifahirozer@gmail.com

*Corresponding author

Received 2015 Sep 11; Accepted 2016 Jan 25

Copyright : @ Surgical Neurology International

This is an open access article distributed under the terms of the Creative Commons Attribution-NonCommercial-ShareAlike 3.0 License, which allows others to remix, tweak, and build upon the work non-commercially, as long as the author is credited and the new creations are licensed under the identical terms.

Abstract

Background:

To identify the role of the hypercholesterolemia as a starting factor in discovertebral degeneration that ultimately causes lower back pain, and investigate the role of Vitamin $\mathrm{E}$ in this process.

Methods:

The rabbits $(n=32)$ were divided into two broad experimental groups: A control group, and a hypercholesterolemia group, namely cholesterol, and cholesterol plus Vitamin E groups and they were fed sequentially for 4 or 8 weeks. Serum cholesterol and Vitamin E ( $\alpha$-tocopherol) levels were determined; vascular tissue was prepared for histopathological analyses and vertebra was decalcified for the study.

Results:

Cholesterol diet group resulted approximately 44-fold of increase plasma cholesterol levels over the 4-week control values. Additional supplementation with Vitamin E group induced a plasma cholesterol level increase of only 37 -fold as compared to the control group. In the cholesterol groups, light microscope examination revealed atherosclerotic plaque in major arteries. However, in the cholesterol plus Vitamin $\mathrm{E}$ treatment groups, no lipid accumulation or foam cell formation was visible in the abdominal aorta and vertebral segmental artery. In histopathological examination, we found degenerative changes in the discovertebral unit in cholesterol treated groups.

Conclusion:

Hypercholesterolemia causes fat accumulation in the disc endplate and vertebral body that causes blood supply disturbances which might be a starting factor of discovertebral degeneration. This event was not reversed by the elimination of cholesterol from the diet. Vitamin $\mathrm{E}$ supplementation was not effective in reducing fat accumulation in vertebral bone marrow. As a result, we conclude that degeneration of the discovertebral unit is not related to atherosclerotic changes in the major blood vessels.

Keywords: Atherosclerosis, disc degeneration, hypercholesterolemia, rabbit spine, spine degenerative disease, Vitamin E

Low back pain resulting from lumbar degenerative disc disease is a common cause of morbidity and deterioration of the quality of life.[26] Many studies have investigated the cause of disc degeneration, and some suggest that intervertebral disc degeneration may result from a variety of factors, including age-related disc changes,[르,28] genetic factors,[] ] and cumulative damage to the spinal segment components as a result of heavy physical work, such as weight lifting or stationary work postures.[14] In 1998, Modic et al. published magnetic resonance imaging data revealing signal changes in vertebral body endplates as a result of hypervascularity (Type I) and fatty replacements of the red bone marrow (Type II).[11] Furthermore, the study established that Modic changes are strongly linked with disc degeneration and are also associated with low back pain.[12,22] On the other hand, some studies have suggested that there is a connection between atherosclerosis and disc degeneration/low back pain.[10] To date, none of the published experimental studies revealed a relationship between hypercholesterolemia and the histological changes in vertebral bone marrow and endplates.

It has been widely accepted that oxidative modification of low-density lipoprotein (LDL) cholesterol plays a pivotal role in the progression of atherosclerosis.[23] Vitamin E has been suggested to aid in protecting against the progression of the disease.[25] The association between hypercholesterolemia and atherosclerosis results in fat accumulation in vertebral bone marrow and endplates, causing diminished blood supply to the vertebrae. This process has received a growing amount of attention as one of the possible underlying factors for spine degenerative disorders. 
In this study, we had two aims. First, we planned to investigate the general effects of hypercholesterolemia on the spine and endplates, specifically focusing on degeneration resulting from fatty replacement. Second, we examined if hypercholesterolemia related changes could be reversed by either Vitamin E or lack of cholesterol in the diet collectively. The study will help us to elucidate the relationship between hypercholesterolemia and atherosclerosis, as well as their mutual impact on spinal degeneration.

\section{Animal model}

Male New Zealand albino rabbits, ranging in age from 2 to 4 months old, were randomly selected as the experimental groups. The animals were maintained and handled in accordance with the Animal Welfare Act and the Guide for the Care and Use of Laboratory Animals prepared by the Animal Ethics Committee of Marmara University. All rabbits were fed 100g of rabbit food per day. Cholesterol was added to the diet as diethyl ether solution. The cholesterol concentration was chosen based on previously reported protocols.[1, 19]

\section{Experimental management}

The rabbits $(n=32)$ were divided into two broad experimental groups: A control group and a hypercholesterolemia group. The control rabbits $(n=8)$ were fed only with Vitamin E-poor diet. The control group was divided into 1a and 1b groups. Four rabbits in Group 1a were fed for 4 weeks and the next four (Group 1b) for 8 weeks without any added supply and treatments. The hypercholesterolemia group rabbits $(n=24)$ were fed with the same Vitamin E-poor diet, except that it was supplemented with 2\% cholesterol. This hypercholesterolemic group was divided into four subgroups (2a, 2b, 2c, and 2d). Rabbits in subgroup 2a $(n=6)$ were fed for 4 weeks. Members of Group 2b $(n=6)$ were fed with the same diet $+2 \%$ cholesterol but also received daily intramuscular injections of $50 \mathrm{mg} / \mathrm{kg}$ of Vitamin E for 4 weeks. The third (2c, $n=$ 6 ) and fourth ( $2 \mathrm{~d}, n=6)$ subgroups were fed with a diet containing $2 \%$ cholesterol for first 4 weeks. Then, the rabbits in Group $2 \mathrm{c}$ received intramuscular Vitamin E (50 mg/kg) injections, while those in Group 2d were fed only with the Vitamin E-poor diet for an additional 4 weeks. After either 4 weeks (in Groups 1a, 2a, and 2b) or 8 weeks (in Groups 1b, 2c, and 2d) the rabbits were subjected to overnight food withdrawal and were subsequently anesthetized using $50 \mathrm{mg} / \mathrm{kg}$ of ketamine hydrochloride. Blood was collected to evaluate Vitamin E and cholesterol levels. The vertebral segmental arteries of all the rabbits were removed for microscopic evaluation. Then a posterior median skin incision was made on the lumbar paravertebral muscles, allowing dissection and complete removal of the lumbar columna vertebralis.

\section{Determination of serum cholesterol and Vitamin E ( $\alpha$-tocopherol)}

Serum cholesterol levels were determined using an automated (Hitachi Modular P800) enzymatic technique (Roche, Boehringer Inelheim).

Vitamin E levels were measured by reverse-phase high-pressure liquid chromatography (RP-HPLC).[19] The $\alpha$-tocopherol ( $\alpha \mathrm{T})$ assay was performed by serum extraction in subdued lighting to produce a $\alpha$ T-rich fraction. Briefly, $1 \mathrm{~mL}$ serum was shaken vigorously in $12 \mathrm{~mL}$ of $9 \%$ $\mathrm{w} / \mathrm{v} \mathrm{KOH}$ in $90 \%$ ethanol $+1 \mathrm{~mL}$ ascorbic acid solution $(10 \% \mathrm{w} / \mathrm{v})$ and incubated at $80^{\circ}$ for $30 \mathrm{~min}$. After cooling, $12 \mathrm{~mL}$ petroleum ether (bp $60-80^{\circ}$ ) was added, then the samples vortexes and centrifuged. The upper organic extract was collected into a new tube, while the aqueous phase was re-extracted with petroleum ether twice more. The organic extracts were pooled, washed 3 times with water, then dried under nitrogen in glass vials and stored at $-80^{\circ} \mathrm{C}$ until they were assayed for $\alpha \mathrm{T}$ by HPLC. Samples were dissolved with $2 \mathrm{~mL}$ methanol by and RPHPLC on a C18 Nova-Pak column (Alltech). The mobile phase was $100 \%$ methanol, and fluorescence detection was performed with excitation at $292 \mathrm{~nm}$ and emission at $350 \mathrm{~nm}$.

\section{Preparation of vascular tissue for histopathological analyses}

The vertebral segmentary arteries from all groups were fixed for histopathology in 10\% buffered paraformaldehyde for a minimum of $24 \mathrm{~h}$. Tissue samples were prepared in an autotechnicon and then embedded in paraffin. The specimens were sectioned $(5 \mu \mathrm{m})$ with a microtome and deparaffinated 3 times with xylene in a $60^{\circ} \mathrm{C}$ incubator. The tissue samples were rehydrated with alcohol, washed with water, and stained with hematoxylin and eosin ( $\mathrm{H}$ and $\mathrm{E}$ ). The morphometric characteristics of tissue samples were evaluated under a light microscope (magnification, $\times 100$ and $\times 400$ ).

\section{Decalcification and preparation of vertebrae}

The specimens were fixed in a $10 \%$ buffered paraformaldehyde for $24 \mathrm{~h}$, and then the osseous parts of the vertebrae were put in a decal solution (Shandon TBD-1, 14\% hydrochloric acid) for another $24 \mathrm{~h}$. The specimen was serially sectioned in sagittal and axial planes and embedded in paraffin. A guillotine was used for the serial sectioning [Figure 1]. The specimens were stained by $\mathrm{H}$ and $\mathrm{E}$ stain.



\section{Figure 1}

Sagittal and axial sections showing the discovertebral unit, which included: (1) disc, (2) disc

endplate, (3) subcondral space, and (4) half of the vertebral bone

\section{Results and statistical analyses}

Cholesterol and Vitamin E serum concentrations Blood analyses for the all experimental groups are shown in Tables $\underline{1}-\underline{3}$. Two percent cholesterol diet supplementation for 4 weeks (Group 2a) resulted in an approximately 44-fold increase in plasma cholesterol levels over the 4week control values (Group 1a). Additional supplementation with Vitamin E (Group 2b) induced a plasma cholesterol increase of only 37-fold relative to the control (Group 1a). Rabbits that were fed on cholesterol for 4 weeks, followed by normal diet + Vitamin E injections for an additional 4 weeks (Group 2c) showed a 15-fold increase in plasma cholesterol levels when compared with the control group (Group 1b) at the end of 8 weeks. Rabbits fed on cholesterol diet for 4 weeks and then with a normal diet for another 4 weeks (Group 2d), showed plasma cholesterol level decrease of 8-fold when compared with Group 2c, which received cholesterol for 4 weeks and Vitamin E for 4 weeks. Furthermore, Group 2d showed a 5-fold increase over control rabbits (Group 1b). Plasma Vitamin E concentrations were higher in the cholesterol-fed rabbits (Group 2a), in direct correlation with the literature data.[13,18] This event is caused by an increased Vitamin E uptake resulting from the enhanced lipid uptake produced by the high cholesterol diet. If Vitamin E were absent from the diet, the effect of cholesterol 
would be more disruptive, and the effect of Vitamin E supplementation would also be exaggerated.

Table 1

Serum concentrations of cholesterol and Vitamin E in all groups

\section{Table 3}

Cholesterol level evaluation of the Groups $1 \mathrm{a}, 2 \mathrm{a}$, and $2 \mathrm{~b}$

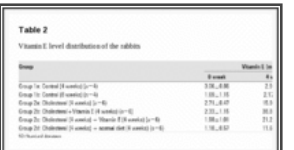

Table 2

Vitamin E level distribution of the rabbits

\section{Statistical analyses}

For the statistical analyses, Number Cruncher Statistical System 2007, Power Analysis and Sample Size 2008 Statistical Software Kaysville, Utah USA were used.

To evaluate the data of the study, descriptive statistical methods as a mean average and standard deviation were referred.

For the comparison of the results of qualitative data of two separate groups exhibiting nonuniform parameters, Mann-Whitney U-test was used.

To compare the qualitative data of nonuniform parameters in the same group, Wilcoxon signed ranks test was used.

Significance level was accepted to be at $P<0.01$ and $P<0.05$.

Interpretation of the statistical result

Evaluation of the results of different groups (1a vs. $2 a$ and $1 a$ vs. $2 b$ ) [Tables $1-3$ ] Considering the initial level of serum cholesterol, there was no significant statistical difference among Groups 1a (fed with normal diet for 4 weeks) and 2a (fed with cholesterol diet for 4 weeks) $(P>0.05)$. This means the two groups were uniform at the beginning, regarding the serum cholesterol levels.

As expected, the cholesterol levels of the Group 1a rabbits were lower than Group 2a, fed with high cholesterol diet at the end of 4 weeks; and this was statistically significant $(P<0.001 ; P<0.01)$.

The initial cholesterol level of the Groups $1 \mathrm{a}$ (fed with a normal diet for 4 weeks) and $2 \mathrm{~b}$ (fed with Vitamin E diet) was statistically insignificant $(P>0.05)$. This also means that these two groups were uniform at the beginning of the study, regarding the serum cholesterol levels.

The cholesterol level of the Group 1a rabbits was lower than the Group $2 \mathrm{~b}$ rabbits at the end of 4 weeks and this was statistically significant (P: $0.001 ; P<0.01$ ), showing that the supplementation with Vitamin $\mathrm{E}$ did not preserve the serum cholesterol profile at the Group $2 \mathrm{~b}$ at the end of the $4^{\text {th }}$ week.

Evaluation of the results within the groups (1a, 2a, and $2 b$ separately) [Tables 1 and 3 ] Decrease of the serum cholesterol level of the Group 1a (fed with a normal diet for 4 weeks) rabbits at the end of the $4^{\text {th }}$ week was found to be statistically insignificant $(P>0.05)$.

Increase of the serum cholesterol level of the Group 2a rabbits (fed with high cholesterol diet for 4 weeks) at the end of 4 weeks was found to be statistically significant (P: $0.001 ; P<0.01)$ as expected.

Increase of the serum cholesterol level of the Group $2 \mathrm{~b}$ rabbits (fed with high cholesterol and Vitamin E diet for 4 weeks) at the end of 4 weeks was found to be statistically significant (P: $0.001 ; P<0.01)$.

Evaluation of the result of different groups ( $1 \mathrm{~b}$ vs. $2 \mathrm{c}$ and $1 \mathrm{~b}$ vs. $2 \mathrm{~d}$ ) [Tables 1 and $\underline{3}$ ] There was no statistically significant difference among the initial cholesterol levels of the Group $1 \mathrm{~b}$ rabbits (fed with a normal diet for 8 weeks) and Group 2c rabbits (fed with high cholesterol diet for the first 4 weeks, and with Vitamin E diet or the next 4 weeks $)(P>0.05)$.

The fourth-week cholesterol level of the Group $1 \mathrm{~b}$ rabbits was statistically significantly lower than those of Group $2 \mathrm{c}(\mathrm{P}: 0.001 ; P<0.01)$.

The 8 weeks cholesterol level of the Group $1 \mathrm{~b}$ rabbits was statistically significantly lower than the Group $2 \mathrm{c}$ rabbits $(\mathrm{P}: 0.001 ; P<0.01)$.

The initial cholesterol levels of the Group $1 \mathrm{~b}$ rabbits (fed with a normal diet for 8 weeks) and $2 \mathrm{~d}$ rabbits (fed with cholesterol diet for 4 weeks and with a normal diet for the next 4 weeks) were statistically similar $(P>0.05)$.

The cholesterol level at the end of the $4^{\text {th }}$ week of Group $1 \mathrm{~b}$ was statistically significantly lower than Group $2 \mathrm{~d}(\mathrm{P}: 0.001 ; P<0.01)$.

The cholesterol level at the end of 8 weeks of Group $1 \mathrm{~b}$ was statistically significantly lower than Group $2 \mathrm{~d}(\mathrm{P}: 0.001 ; P<0.01)$.

Evaluation of the results within the Groups $1 \mathrm{~b}, 2 \mathrm{c}$, and $2 \mathrm{~d}$ separately [Tables 1 and 4$]$

Table 4
Cholesterol level evaluation of the Groups $1 \mathrm{~b}, 2 \mathrm{c}$, and $2 \mathrm{~d}$

Decrease of the cholesterol level of the Group $1 \mathrm{~b}$ at the end of the $4^{\text {th }}$ week, according to the initial level was found to be statistically insignificant $(P>0.05)$. Either, the increase of the cholesterol level of this group at the end of the 8 weeks, according to the initial level was found to be insignificant $(P>0.05)$.

Considering the Group 2c rabbits, fed with cholesterol diet for the initial 4 weeks, and with Vitamin E diet for the next 4 weeks, the increase of the cholesterol level at the end of the $4^{\text {th }}$ week, according to the initial level was found to be statistically significant $(\mathrm{P}: 0.001 ; P<0.01)$. 
Besides, the increase of the cholesterol level of this group at the end of the 8 weeks, according to the initial level was found to be statistically significant (P: 0.001; $P<0.01)$.

The increase of the $4^{\text {th }}$ week cholesterol level according to the initial level of the Group $2 \mathrm{~d}$ rabbits, fed with cholesterol diet at the first 4 weeks, and with normal diet at the next 4 weeks was found to be statistically significant (P: $0.001 ; P<0.01$ ).

The increase in the cholesterol level of this group at the end of 8 weeks was found to be statistically significant (P: $0.012 ; P<0.05)$.

\section{Histopathological analyses of vascular tissue}

Rabbit abdominal aortas and vertebral segmental arteries from the different experimental groups were studied. Representative light microscopy images of the vertebral segmental artery from control rabbits [Figure 2] (Group 1a), and rabbits from the cholesterol Group 2a, showed atherosclerotic vascular with lipid deposits in the subendothelial and medial layers, degeneration of elastic fibers in the medial layer, endothelial desquamation, and decrease of wave in the endothelial layer. Tissue specimens of the rabbits from the cholesterol plus Vitamin E treatment group $(2 \mathrm{~b}, 2 \mathrm{c})$ showed no lipid accumulation or foam cell formation (magnification, $\times 400$ ) [igure 2]. Thus, Vitamin E reduces lipid accumulation, resulting in fewer cholesterol plaques in vertebral segmental arteries.

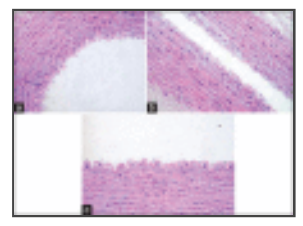

\section{Figure 2}

Representative light microscopy images of the rabbit segmental artery from: (a) control rabbits, (b) rabbits from the cholesterol group, which showed atherosclerotic vascular with foam cells in the subendothelial and medial layers and endothelial desquamation ...

Histopathological analyses of discs, disc endplates, and vertebras

In the control group, normal histological findings in the annulus and endplate, along with normal neural elements of the spinal cord and nerve roots were observed. A discovertebral unit is comprised of vertebral bone marrow, disc endplates, and the disc component. In rabbits from the control groups, the endplate was composed of an osseous component as well as hyaline cartilage material. The disc endplate is considered to be the thin layer of hyaline cartilage that lies between the bone of the vertebral body and the soft tissue of the disc. In rabbits, the disc endplates are typically $<0.1 \mathrm{~mm}$ [ Figure 3].

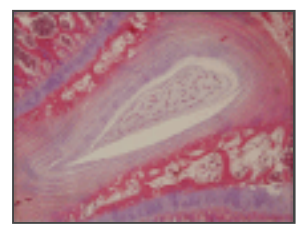

\section{Figure 3}

Control group, normal histological findings in the disc endplate $(0.1 \mathrm{~mm})$ is considered to be the thin layer of hyaline cartilage that lies between the bone of the vertebral body and the soft tissue of the disc $(\times 40)$

In our histopathological examination, we found degenerative changes in the discs, disc endplates, and vertebral bodies of the cholesterol treated rabbits for 4 weeks. Accumulation of foam cells (lipid deposits) in the disc endplates and bone marrow caused disruption of the blood supply. This process could mark the beginning of degenerative changes in disc endplates. In addition to this finding, there were solitary or aggregated foamy histiocytes (xanthic cells) in the cartilage, bone marrow, tendons, and soft tissue [Figure 4a] .

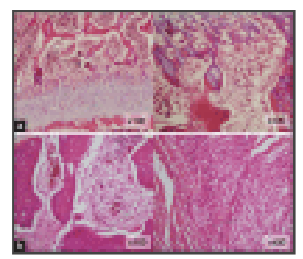

Figure 4

Histopathological examination; (a) 2\% cholesterol feed group $(\times 100)$ and (b) cholesterol plus Vitamin E treatment group demonstrated degenerative changes in the discs, disc endplates and vertebral bodies, accumulation of foamy histiocytes (xanthic ...

In rich cholesterol-fed plus Vitamin E-supplement groups for 4 weeks, histopathological examination revealed changes in the discovertebral unit that were similar to those in the rich cholesterol-fed groups. We did not find any discernible decreases in the number of foam cells related to Vitamin E, whereas Vitamin E certainly had a significant effect on atherosclerotic lesions in the large arteries of these same rabbits. Therefore, we concluded that Vitamin E was insufficient to diminish foam cells, despite its effect on atherosclerotic lesions in large vessels for this treatment period [Figure $4 \mathrm{~b}]$.

In rich cholesterol-fed plus Vitamin E-supplemented groups for 8 weeks (first 4 weeks rich cholesterol + second 4 weeks Vitamin E supplement), histopathological examination did not reveal discernible decreases in the number of foam cells in spite of the supplementation of Vitamin E on second 4 weeks. In rich cholesterol-fed and normal diet groups for 8 weeks ( first 4 weeks rich cholesterol + second 4 weeks normal diet), we observed no changes in discovertebral fat accumulation on second 4 week. We conclude that hypercholesterolemia causes degenerative changes in the discovertebral unit [igure 5].

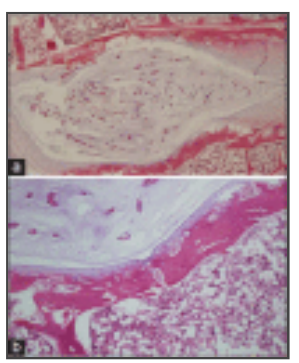

\section{Figure 5}

Histopathological examination; rich cholesterol-fed plus Vitamin E-supplement groups for 8 weeks $(\times 400)(a)$, rich cholesterol-fed and normal diet groups for 8 weeks. We observed no diminish in discovertebral fat accumulation at the end of 8 weeks ... 
segmental. Lumbar ischemia may have several different outcomes, affecting structures such as nerve roots, vertebral bodies, intervertebral discs, and paravertebral muscles. Depending on the structures nourished by blood, symptoms may vary: Ischemia of the vertebral bone may cause continuous dull pain, typical of skeletal ischemia. Nerve root ischemia may evoke radicular pain. The intervertebral disc is supplied by diffusion through the endplates from vertebral bodies above and below it. Furthermore, the disc is located at the end of oxygen and nutrient chain, making it the first structure to suffer during nutrient insufficiency.[10]

Hypercholesterolemia plays an important role in the progression of atherosclerosis.[4,27] Severe hypercholesterolemia is shown to cause macrophage infiltration through vascular endothelium and this in turn results in atherosclerotic plaque aggregation and vascular lesions in

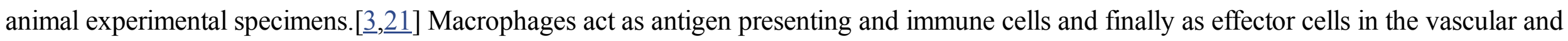
synovial and cartilage tissues. They in turn cause hyperplasia of the vascular lining layer, mononuclear infiltration, cartilage destruction, and are the major precursors of the inflammatory mediators. When activated, they also differentiate into osteoclast-like cells, causing bone resorption. Hyperlipidemia is also suggested to induce osteoclastic potential of the cells in bone marrow in vivo. Lipid oxidation products and osteoclast cell enlargement in bones were documented in hyperlipidemic mice.[24] Moreover, diet-induced cholesterol increase was shown to be associated with reduced bone quality, similar to osteoporosis. Hence, hypercholesterolemia may be a starting factor of bone quality decrease and cartilage and bone degeneration of both the vertebral column and the vascular structures through the activation of the mononuclear phagocytic system.[21]

As the pathophysiological mechanisms of inflammation are similar in vascular tissue (atherosclerosis) and cartilage tissue, we assume that hypercholesterolemia may contribute to intervertebral disc degeneration by the same mechanism of inflammation and by affecting the vessels supplying the disc tissue. The damaged synovial membrane, calcified cartilage, neighboring bone tissue, and angiogenesis in turn may lead to the vertebral body Modic degeneration and at the same time, causing prominent atherosclerosis in the aorta and vessels nourishing the vertebral column.

The protective effects of antioksidants such as $\alpha \mathrm{T}$ (Vitamin E) on bone metabolism have been studied in animals and humans, and these studies show generally the beneficial effects of this metabolite on bone metabolism. There are a number of studies in the literature about the relationship between hypercholesterolemia, $\alpha \mathrm{T}$ (Vitamin E) supplementation and bone and cartilage metabolism, and vascular changes. These studies try to focus on the alterations of the histologic structure of vascular, osseous, and cartilage tissue caused by cholesterol increase and whether these alterations can be reversed by supplementing Vitamin E.

Kasai et al. studied the effect of $\alpha \mathrm{T}$ on the vertebral and femur bone of ovariectomized rats. They found that $\alpha \mathrm{T}$ supplementation caused a tendency for the bone mass increase and remodeling of the vertebral body in normal rats.[] $]$

Hampson et al. studied $\alpha \mathrm{T}$ relationship with bone metabolism and arterial stiffness in postmenopausal women and they concluded that high serum concentrations of $\alpha \mathrm{T}$ might have a negative effect on bone formation. [ㅁ]

The effect of hypercholesterolemia on synovial and vascular endothelial structure has been discussed in the literature. Similar to our study, Prieto-Potín et al. induced hypercholesterolemia in rabbits by administrating high-fat diet and analyzed lipid and systemic markers in blood serum. We can assume that vertebral unit is a kind of joint structure, composed of bone (vertebral body), cartilage (end plate and disc tissue), and synovium (lining facet cartilage), and the results of Potin's study could be simulated to our study, as they examined knee joint.

Hypercholesterolemia causes cartilage-bone damage, and foam macrophages are the main causes in this process. Inflammatory actions induced by adipokines explain some of the associations between skeletal and cardiovascular effects of hypercholesterolemic cases. The biochemical causes of disc-cartilage inflammation and their vascular adverse effects may be related to cytokines, chemokines, adipokines such as tumor necrosis factor-alpha, interleukin-6, plasminogen activator inhibitor, end others. Endothelial dysfunction is a result of these chemokines and inflammatory molecules, causing increased vessel permeability. Leukocytes rush toward the synovia, cartilage. Lipid infiltration, foam cell, and fatty streaks within the vessel wall, eventually cause atherosclerotic plaques. Mainly, macrophage infiltration promotes atherosclerosis and also causes cartilage changes. Macrophages act as antigen presenting cells and finally cause inflammation in acute and chronic cases.

Besides, macrophages may be transformed to cathepsin K-stained osteoclasts and cathepsin $\mathrm{K}$ is the major osteoclast protease responsible for bone resorption. Thus, this metabolic event in hyperlipidemia causes bone degeneration. A terminal degradation product of these osteoclasts, C-terminal telopeptide of Type I collagen may be used as an indicator of bone resorption, degeneration and also it is found in atherosclerotic plaques.[리, $\underline{24}]$

The mononuclear cells infiltrating to the vascular wall and cartilage differentiate to osteoclast-like cells and cause bone resorption. This aggressive infiltration eventually causes degenerative changes in the joints, vertebral bone, and vascular tissue.

Bone quality parameters are altered by a hypercholesterolemic diet in animal experimental studies, including bone volume fraction, bone mineral density, trabecular number, alterations in mechanical properties of the bone, and an increase in osteoclast number.[20] In the study of Okayasu et al., it has been shown that LDL level decrease causes impaired osteoclastogenesis and a subsequent decrease of bone resorption parameters and increased bone mass in mice.[16] Thus, we may conclude that hypercholesterolemia directly disturbs homeostasis of the vertebral bone structure.[21]

Hyperlipidemia increases systemic inflammatory effects on the vascular bone and cartilage tissues by causing massive macrophage infiltration and transformation to foam cells and osteoclasts and in turn leading to atherosclerosis and disc and vertebral degeneration.

The role of $\alpha \mathrm{T}$ (Vitamin E) has been studied in hypercholesterolemia as an antioxidant, primarily for preventing the vascular effects. Vitamin E supplementation may be associated with a lower risk of atherosclerosis and lower risk of coronary heart disease, but it is dose dependent. Highdose Vitamin E supplementation (over $400 \mathrm{IU} /$ day) may be associated with an increased mortality rate. Besides the vascular effects, osteogenic effects of Vitamin $\mathrm{E}$ have been studied widely. Vitamin E is shown to cause bone mass increase in cancellous bones of the vertebral body, leading to beneficial effects on bone health. The osteoclast activation of free radicals, affecting bone turnover is reversed in the benefit of bone remodeling by antioxidants as Vitamin E. The underlying mechanism has not been determined thoroughly yet.[]]

In this study, we evaluated the effect of a hypercholesterolemia as a starting factor on spinal degeneration in an experimental animal model 
(rabbits) and the role of Vitamin E. A high cholesterol supplementation was preferred to lower cholesterol-containing diets (5-10 g/kg). This decision turned out to have the advantage of maximizing the atherosclerotic result and diminishing the treatment time as a result elevation in blood cholesterol in rabbits far exceed that seen in the most humans. Progressive degeneration of the disc endplates and changes to the vertebral body were found to be correlated with high blood concentrations of cholesterol. The noticeable concession of the current study, when compared to similar studies is that not only the effect of hypercholesterolemia and Vitamin $\mathrm{E}$ on the major vascular tissue and on the discovertebral unit was investigated, but also serum concentration, vascular and discovertebral unit histological changes were examined at the same study. One limitation of the study was that it encompassed a relatively short period of investigation (4 and 8 weeks). However, the length of the time period was proved to be sufficient for revealing vascular changes, such as plaque formation, which is associated with hypercholesterolemia and which was congruent with the time frame of the previous publications in the literature. $[17, \underline{18}]$ Furthermore, causes of discovertebral unit changes have already been discovered, but we believe that the current study calls attention to discovertebral degeneration associated with high serum levels of cholesterol. Chronic changes as well as the latter stages of discovertebral degeneration might have been revealed in a longer lasting study.

Disc degeneration is a very complex phenomenon that will require extensive biomechanical and biochemical researches before it will be fully understood.[14] Many studies have investigated the relationships between the biomechanical performance of the discovertebral unit and degeneration.[6] These biomechanical studies revealed that high serum levels of cholesterol cause accumulation of fat at the discovertebral unit, particularly in the disc endplate. This accumulation causes vascular disorders and accelerates the ischemia in the discovertebral unit that causes degeneration.

Atherosclerosis of aorta and stenosis of the lumbar spine feeding arteries are all proven to be associated with degenerative disc disease. Cardiovascular risk factors of atherosclerosis on the other hand are also proven by many studies.

The discovertebral junction is a dynamic zone that demonstrates normal variations and can be modified by disease, which makes biomechanical and biochemical investigations inevitable in the study of degeneration. Possible support for the association between endplate degeneration and changes in subchondral bone and bone marrow include histological specimens from groups that were given food containing $2 \%$ cholesterol. As a consequence of high cholesterol diet increased oxidative stress and vascular smooth muscle cell proliferation may initiate, release of inflammatory parameters and other locally liberated factors and has been assumed to be important in the development of vascular and discovertebral unit histological changes.[18, 19$]$ According to our hypothesis, the histological changes in the discovertebral unit as a result of high blood concentrations of cholesterol could play a major role in initiating the degeneration process.

Vitamin E $(\alpha \mathrm{T})$ has been shown to regulate a number of cellular processes, including signal transduction and cell proliferation.[15, 17, 29] Due to its anti-proliferative, anti-inflammatory properties, Vitamin $\mathrm{E}$ has been shown to play a role in protecting against the progression of atherosclerosis, caused by a high cholesterol diet, and its role has been studied in hypercholesterolemia as an antioxidant, primarily for preventing the vascular effects. Vitamin E supplementation may be associated with a lower risk of atherosclerosis and lower risk of coronary heart disease, but it is dose dependent. High-dose Vitamin E supplementation (over 400 IU/day) may be associated with an increased mortality rate. Besides the vascular effects, osteogenic effects of Vitamin $\mathrm{E}$ has been studied widely. Vitamin $\mathrm{E}$ is shown to cause bone mass increase in cancellous bones of the vertebral body, leading to beneficial effects on bone health. The osteoclast activation of free radicals, affecting bone turnover is reversed in the benefit of bone remodeling by antioxidants as Vitamin E. The underlying mechanism has not been determined thoroughly yet.[] $]$

A high plasma concentration of cholesterol, in particular high levels of LDL, is one of the principal risk factors for atherosclerosis. The lesions occur in muscular arteries and can lead to ischemia of the organs that are supplied by the atherosclerotic artery. Necropsy studies have demonstrated a marked association between atheromatous lesions in the aorta and lumbar disc degeneration and stenosis of the feeding arteries of the lumbar vertebrae. These changes in turn lead to lumbar back pain.[10] Lower back pain as a result of discovertebral unit degeneration and atherosclerosis has been the subject of intense research. One necropsy study suggested an association between decreased blood supply of the lumbar vertebrae and lower back pain as a result of discovertebral unit degeneration.[9] These studies provide a solid support for the notion that there is a relationship between large arteries, such as the aorta, and lower back problems as a result of discovertebral unit degeneration. Results of the current study indicate that hypercholesterolemia causes fat accumulation in the disc tissue, endplate, and vertebral body leading to tissue destruction caused by diminished blood supply. In fact, this is an inflammatory process. Our histological examination revealed a significant reduction in markers of atherosclerosis in the Vitamin E supplement groups. However, there were no significant changes in the degenerative process of the discovertebral unit. We concluded that the initiation of discovertebral unit degeneration is directly related to the accumulation of fat tissue in the disc endplate and vertebral bone marrow. It may set off degeneration of the disc itself. Although Vitamin $\mathrm{E}$ supplementation was not effective in reducing fat accumulation in the vertebral bone marrow, this dietary intervention diminished atherosclerotic changes in the same group. Despite the reduction in atherosclerotic plaque and the consequent restoration of blood flow to the discovertebral unit, we observed no changes in discovertebral fat accumulation.

Besides, the lack of effectiveness of the Vitamin E in preventing the discovertebral degeneration may be due to the duration of the experiment (8 weeks). Vitamin E might be effective in preventing the discovertebral degeneration in a longer time period. On the other hand, vascularization of vertebral body is weak, particularly disc tissue has no vessels, but aorta is rich in vasa vasorum. According to our opinion, consequent researches are needed about this issue.

As a result, we conclude that degeneration of the discovertebral unit is not related to atherosclerotic changes in the large blood vessels, such as the aorta or spine segment artery.

Further experimental and prospective clinical studies are needed to clarify the association of atherosclerosis and low back disorders, disc and vertebral degeneration and the precautions to reverse this pathology. 
Conflicts of Interest

There are no conflicts of interest.

Footnotes

http://surgicalneurologyint.com/Effect-of-a-hypercholesterolemia-as-a-starting-factor-on-spinal-degeneration-in-rabbits-and-role-of-Vitamin-E-(a-tocopherol)/

1. Bocan TM, Mueller SB, Mazur MJ, Uhlendorf PD, Brown EQ, Kieft KA. The relationship between the degree of dietary-induced hypercholesterolemia in the rabbit and atherosclerotic lesion formation. Atherosclerosis. 1993;102:9-22. [PubMed]

2. Boos N, Weissbach S, Rohrbach H, Weiler C, Spratt KF, Nerlich AG. Classification of age-related changes in lumbar intervertebral discs: 2002 Volvo Award in basic science. Spine (Phila Pa 1976) 2002;27:2631-44. [PubMed]

3. Cosar M, Iplikcioglu AC, Aytan N, Ozcan D, San T, Kartal-Ozer N, et al. The effect of temporary aneurysm clip on the common carotid artery of atherosclerotic rabbits. Surg Neurol. 2008;69:483-8. [PubMed]

4. Crowther MA. Pathogenesis of atherosclerosis. Hematology Am Soc Hematol Educ Program. 2005;1:436-41. [ubMed]

5. Hampson G, Edwards S, Sankaralingam A, Harrington DJ, Voong K, Fogelman I, et al. Circulating concentrations of Vitamin E isomers: Association with bone turnover and arterial stiffness in post-menopausal women. Bone. 2015;81:407-12. [PubMed]

6. Hsieh AH, Hwang D, Ryan DA, Freeman AK, Kim H. Degenerative anular changes induced by puncture are associated with insufficiency of disc biomechanical function. Spine (Phila Pa 1976) 2009;34:998-1005. [PubMed]

7. Karppinen J, Daavittila I, Solovieva S, Kuisma M, Taimela S, Natri A, et al. Genetic factors are associated with modic changes in endplates of lumbar vertebral bodies. Spine (Phila Pa 1976) 2008;33:1236-41. [PubMed]

8. Kasai S, Ito A, Shindo K, Toyoshi T, Bando M. High-dose $\alpha$ tocopherol supplementation does not induce bone loss in normal rats. PLoS One. 2015;10:e0132059. [PMC free article] [PubMed]

9. Kauppila LI, Tallroth K. Postmortem angiographic findings for arteries supplying the lumbar spine: Their relationship to low-back symptoms. J Spinal Disord. 1993;6:124-9. [PubMed]

10. Kauppila LI. Atherosclerosis and disc degeneration/low-back pain - A systematic review. Eur J Vasc Endovasc Surg. 2009;37:661-70. [PubMed]

11. Modic MT, Steinberg PM, Ross JS, Masaryk TJ, Carter JR. Degenerative disk disease: Assessment of changes in vertebral body marrow with MR imaging. Radiology. 1988;166(1 Pt 1):193-9. [uued]

12. Kjaer P, Korsholm L, Bendix T, Sorensen JS, Leboeuf-Yde C. Modic changes and their associations with clinical findings. Eur Spine J. 2006;15:1312-9. [PMC free article] [PubMed]

13. Morel DW, de la Llera-Moya M, Friday KE. Treatment of cholesterol-fed rabbits with dietary Vitamins $\mathrm{E}$ and C inhibits lipoprotein oxidation but not development of atherosclerosis. J Nutr. 1994;124:2123-30. [PubMed]

14. Natarajan RN, Williams JR, Andersson GB. Recent advances in analytical modeling of lumbar disc degeneration. Spine (Phila Pa 1976) 2004;29:2733-41. [PubMed]

15. Negis Y, Aytan N, Ozer N, Ogru E, Libinaki R, Gianello R, et al. The effect of tocopheryl phosphates on atherosclerosis progression in rabbits fed with a high cholesterol diet. Arch Biochem Biophys. 2006;450:63-6. [PubMed]

16. Okayasu M, Nakayachi M, Hayashida C, Ito J, Kaneda T, Masuhara M, et al. Low-density lipoprotein receptor deficiency causes impaired osteoclastogenesis and increased bone mass in mice because of defect in osteoclastic cell-cell fusion. J Biol Chem. 2012;287:19229-41. [PMC free article] [PubMed]

17. Ozer NK, Azzi A. Effect of Vitamin E on the development of atherosclerosis. Toxicology. 2000;148:179-85. [PubMed]

18. Ozer NK, Negis Y, Aytan N, Villacorta L, Ricciarelli R, Zingg JM, et al. Vitamin E inhibits CD36 scavenger receptor expression in hypercholesterolemic rabbits. Atherosclerosis. 2006;184:15-20. [PubMed]

19. Ozer NK, Sirikçi O, Taha S, San T, Moser U, Azzi A. Effect of Vitamin E and probucol on dietary cholesterol-induced atherosclerosis in rabbits. Free Radic Biol Med. 1998;24:226-33. [릴ed]

20. Pelton K, Kreider J, Joiner D. Hypercholesterolemia promotes an osteoporotic phenotype. Am J Pathol. 2015;15:1-9. [MC free article] [PubMed]

21. Prieto-Potín I, Roman-Blas JA, Martínez-Calatrava MJ, Gómez R, Largo R, Herrero-Beaumont G. Hypercholesterolemia boosts joint destruction in chronic arthritis. An experimental model aggravated by foam macrophage infiltration. Arthritis Res Ther. 2013;15:R81. [PMC free article] [PubMed]

22. Solgaard Sorensen J, Kjaer P, Jensen ST, Andersen P. Low-field magnetic resonance imaging of the lumbar spine: Reliability of qualitative evaluation of disc and muscle parameters. Acta Radiol. 2006;47:947-53. [PubMed]

23. Steinberg D, Parthasarathy S, Carew TE, Khoo JC, Witztum JL. Beyond cholesterol. Modifications of low-density lipoprotein that increase its atherogenicity. N Engl J Med. 1989;320:915-24. [PubMed]

24. Tintut Y, Morony S, Demer LL. Hyperlipidemia promotes osteoclastic potential of bone marrow cells ex vivo. Arterioscler Thromb Vasc 


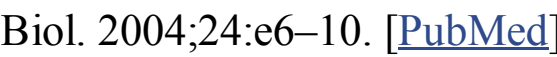

25. Upston JM, Kritharides L, Stocker R. The role of Vitamin E in atherosclerosis. Prog Lipid Res. 2003;42:405-22. [ubMed]

26. Waddell G. Low back pain: A twentieth century health care enigma. Spine (Phila Pa 1976) 1996;21:2820-5. [PubMed]

27. Weber C, Noels H. Atherosclerosis: Current pathogenesis and therapeutic options. Nat Med. 2011;17:1410-22. [PubMed]

28. Zhao CQ, Wang LM, Jiang LS, Dai LY. The cell biology of intervertebral disc aging and degeneration. Ageing Res Rev. 2007;6:247-61. [PubMed]

29. Zingg JM, Azzi A. Non-antioxidant activities of Vitamin E. Curr Med Chem. 2004;11:1113-33. [PubMed] 
Copyright of Surgical Neurology International is the property of Medknow Publications \& Media Pvt. Ltd. and its content may not be copied or emailed to multiple sites or posted to a listserv without the copyright holder's express written permission. However, users may print, download, or email articles for individual use. 